\title{
Electrospun PEDOT:PSS/PVP Nanofibers for CO Gas Sensing with Quartz Crystal Microbalance Technique
}

\author{
Hong-Di Zhang, ${ }^{1,2}$ Xu Yan, ${ }^{1}$ Zhi-Hua Zhang, ${ }^{1}$ Gu-Feng Yu, ${ }^{1}$ \\ Wen-Peng Han, ${ }^{1,2}$ Jun-Cheng Zhang, ${ }^{1,2}$ and Yun-Ze Long ${ }^{1,2,3}$ \\ ${ }^{1}$ College of Physics, Qingdao University, Qingdao 266071, China \\ ${ }^{2}$ Key Laboratory of Photonics Materials and Technology in Universities of Shandong, Qingdao University, Qingdao 266071, China \\ ${ }^{3}$ Collaborative Innovation Center for Marine Biomass Fibers, Materials \& Textiles of Shandong Province, \\ State Key Laboratory Cultivation Base of New Fiber Materials \& Modern Textile, Qingdao University, Qingdao 266071, China
}

Correspondence should be addressed to Hong-Di Zhang; hongdi_zhang@163.com and Yun-Ze Long; yunze.long@163.com

Received 8 December 2015; Revised 20 March 2016; Accepted 7 April 2016

Academic Editor: Chalongrat Daengngam

Copyright (C) 2016 Hong-Di Zhang et al. This is an open access article distributed under the Creative Commons Attribution License, which permits unrestricted use, distribution, and reproduction in any medium, provided the original work is properly cited.

\begin{abstract}
Poly(3,4-ethylenedioxythiophene):poly(styrenesulfonate)/polyvinylpyrrolidone (PEDOT:PSS/PVP) composite nanofibers were successfully fabricated via electrospinning and used as a quartz crystal microbalance (QCM) sensor for detecting CO gas. The electrical property of individual PEDOT:PSS/PVP nanofibers was characterized and the room temperature resistivity was at the magnitude of $10^{5} \Omega \cdot \mathrm{m}$. The QCM sensor based on PEDOT:PSS/PVP nanofibers was sensitive to low concentration (5-50 ppm) CO. In the range of 5-50 ppm CO, the relationship between the response of PEDOT:PSS nanofibers and the CO concentration was linear. Nevertheless, when the concentration exceeded $50 \mathrm{ppm}$, the adsorption of the nanofiber membrane for CO gas reached saturation and the resonant frequency range had no change. Therefore, the results open an approach to create electrospun PEDOT:PSS/PVP for gas sensing applications.
\end{abstract}

\section{Introduction}

Carbon monoxide (CO) is highly toxic, combustible, and explosive. Therefore it is of great importance to notice its leakage in its former stage and give effective monitoring to avoid any major accidents. Although much attention was paid to metal oxide gas sensor materials such as $\mathrm{ZnO}, \mathrm{SnO}_{2}$, and $\mathrm{TiO}_{2}$, in order to achieve higher sensitivity, the normal work temperature of these metal oxide-based gas sensors is rather high $\left(>200^{\circ} \mathrm{C}\right)[1-4]$. Gratifyingly conducting polymer acts as gas sensor material that can work at room temperature to solve the problems.

Conducting polymers possess many interesting properties, such as controllable conductivity, stability, and biocompatibility. Recently, conducting polymers, such as polyaniline (PANI), polypyrrole (PPY), and polythiophene, have become attractive materials for gas sensing application [5-8]. Poly(3,4-ethylenedioxythiophene) (PEDOT) is one of the most promising conducting polymers due to its high conductivity and excellent environmental stability [9]. Although PEDOT itself cannot be easily cast into films due to its insoluble property, PEDOT doped with poly(styrenesulfonate) (PSS) can be dispersed well in the aqueous solution. PEDOT:PSS complex is a blend of oxidatively doped, cationic, conjugated polythiophene derivation (PEDOT) and excess quasi-insulating PSS. The gas sensing properties of PEDOT:PSS nanostructures are investigated mostly by impedance test. PEDOT:PSS films have been used as sensing material for the detection of NO [5] and $\mathrm{NH}_{3}$ [10]. Electrospun PEDOT:PSS/polyvinylpyrrolidone (PEDOT:PSS/PVP) nanofibers have been used to measure the gas sensing to organic gases such as ethanol, methanol, and acetone usually by an electric resistance measurement method [11]. In addition, metal oxide fibers coated with PEDOT:PSS can be used as gas sensor at room temperature by measuring the impedance $[6,12]$.

Except the common impedance method, there is considerable interest in piezoelectric sensors known as quartz 


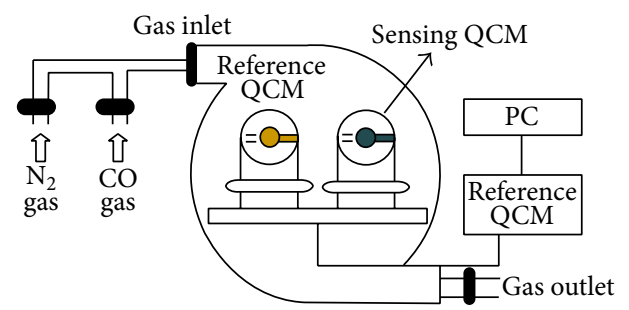

FIGURE 1: Schematic illustration of the experimental apparatus as QCM sensor.

crystal microbalance (QCM) due to its high mass sensitivity. Zeng et al. [13] used the QCM method to measure the PEDOT:PSS sensitivity to organophosphorus pesticide o,odimethyl-o-2,2-dichlorovinyl phosphate (DDVP). However, to the best of our knowledge, the $\mathrm{CO}$ sensing characteristics of the PEDOT:PSS nanofibers using a QCM sensing platform have not been reported yet. In this paper, conducting polymer PEDOT:PSS/PVP composite nanofibers were prepared by electrospinning and collected on the QCM. Aligned nanofibers were electrospun and the electrical resistivity of individual nanofibers was measured. The gas sensing property of the PEDOT:PSS/PVP nanofibers was investigated for detection of CO gas using QCM sensing platform.

\section{Experimental}

2.1. Preparation of Aligned PEDOT:PSS/PVP Nanofibers. $1.8 \mathrm{~g}$ PVP powder was added into $8.2 \mathrm{~g}$ of ethanol (AR, Tianjin Damao Chemical Reagent Factory) and stirred to form uniform and transparent solution. Then $1.1 \mathrm{~g}$ PEDOT:PSS was mixed into the previous PVP solution and stirred for two hours. Then the precursor solution was obtained. Firstly, to measure the electrical property of individual PEDOT:PSS nanofiber, aligned nanofibers were prepared via centrifugal electrospinning. The centrifugal electrospinning setup has been reported in detail [14]. The solution was loaded into $5 \mathrm{~mL}$ plastic syringe with stainless steel needle connected to a high-voltage power supply (Tianjin Dongwen HighVoltage Power Supply Plant). The voltage for centrifugal electrospinning was $1.6 \mathrm{kV}$. The distance between the tip of the needle and collector was $4.0 \mathrm{~cm}$. The rotating speed was $420 \mathrm{rpm}$, and the parallel nanofibers were collected on the aluminum foil.

2.2. Preparation of PEDOT:PSS/PVP and PVP Gas Sensor. PEDOT:PSS/PVP nanofiber membrane was prepared by traditional electrospinning. The above precursor solution was loaded into a $5 \mathrm{~mL}$ plastic syringe with a stainless steel needle. The voltage was $20 \mathrm{kV}$. The distance between the tip of the needle and collector was $10 \mathrm{~cm}$. The pure PVP nanofibers were also prepared by traditional electrospinning as follows: $1.8 \mathrm{~g}$ PVP powder was mixed into $8.2 \mathrm{~g}$ of ethanol to form the PVP precursor. And then the precursor solution was electrospun under an applied voltage of $20 \mathrm{kV}$ and an electrospinning distance of $10 \mathrm{~cm}$. The QCM (CHI400C, Shanghai Huachen Instruments Company Limited) was set on the aluminum foil as collector to attach nanofibers.
After electrospinning for one minute, the high-voltage power supply was turned off, and the QCM coated with nanofiber membrane was taken out. And then the QCM was heated at $60^{\circ} \mathrm{C}$ for two hours. This QCM was the sensitive component of gas sensor.

2.3. Measurement Setup. The gas sensor measurements were carried out in a closed gas chamber (Figure 1). Two QCM electrodes were set in the gas chamber: one is the reference QCM which is blank and the other is coated with sensing nanofiber membrane, which were connected with frequency meter. The theory of QCM was based on piezoelectric effect: QCM produced vibrations under certain voltage. The mass of QCM with membrane changed when the sensing materials interacted with the measured gas, which led to AC electric field changing and the vibration frequency of QCM changing. Therefore, the oscillation frequencies of the two QCM electrodes were recorded and treated by frequency meter. Then the frequency difference between the two QCMs was obtained and output to a computer.

All experiments were performed at room temperature. The gas sensing measurements included two processes: firstly, $\mathrm{N}_{2}$ gas was filled into the sealed gas chamber at a flow rate of $500 \mathrm{sccm}$ until the frequency difference between the reference QCM and the sensing QCM was stable at $0 \mathrm{~Hz} \pm$ $1 \mathrm{~Hz}$. Then $\mathrm{N}_{2}$ gas was closed and $\mathrm{CO}$ was inlet at a flow rate of $500 \mathrm{sccm}$. The sensor interacted with $\mathrm{CO}$ which led to the mass changing and frequency changing. When the frequency difference was stable again, $\mathrm{N}_{2}$ gas was filled into the chamber again until the output frequency regained its stability.

The morphologies of the nanofibers were characterized by a scanning electron microscope (SEM, JEOL JSM-6390). The electrical property was performed by a two-metalmicroprobe testing platform (HP 4156A precision semiconductor parameter analyzer). Raman spectra were recorded in air at room temperature by means of a LabRAM Infinity spectrometer (Raman, Jobin Yvom S.A.S.).

\section{Results and Discussion}

3.1. Morphology, Structure, and Electrical Property. Single nanofiber was used to measure electrical conductivity of PEDOT:PSS/PVP, which was closer to intrinsic electrical characteristics of the nanofibers than that of nonwoven fibers. Moreover, to measure electrical properties, drawing individual nanofiber from nanofiber arrays can be easily performed compared with the crossed structures of the nonwoven fibers. 


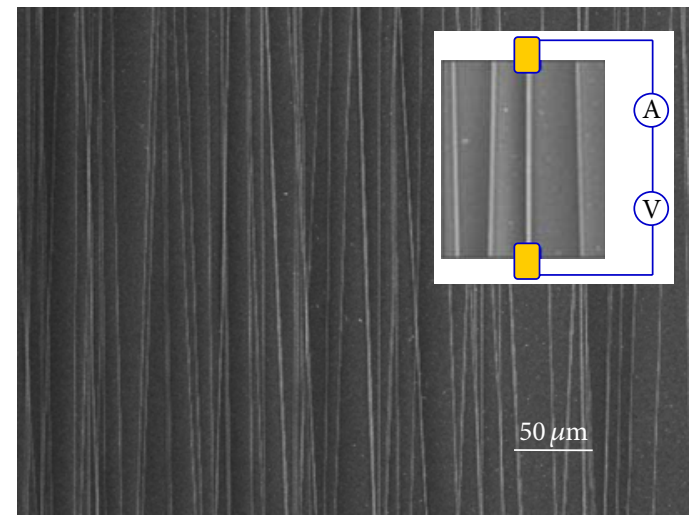

(a)

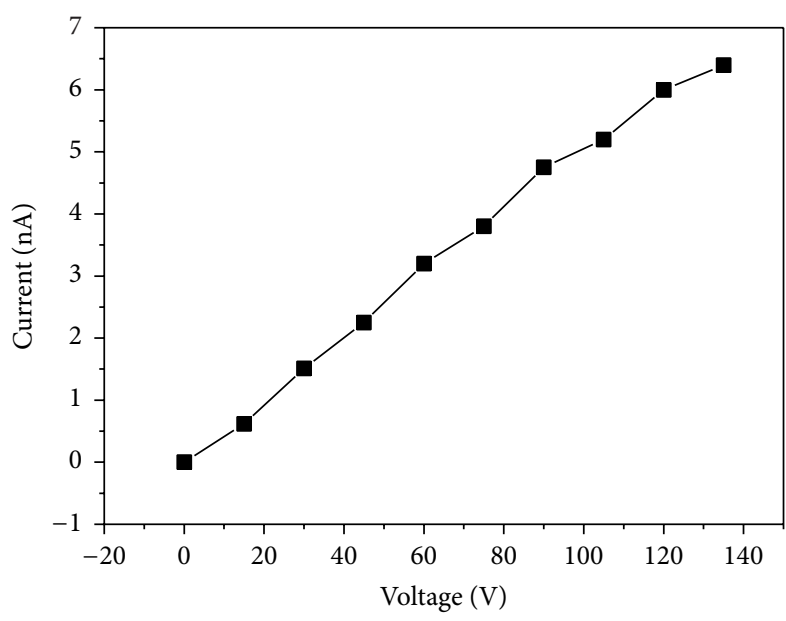

(b)

FIGURE 2: (a) SEM image of the aligned PEDOT:PSS/PVP nanofibers prepared by centrifugal electrospinning. The smaller picture displays schematic illustration of electrical conductivity measurement of isolated nanofibers by a two-metal-microprobe method. (b) $I-V$ curve of isolated fibers.

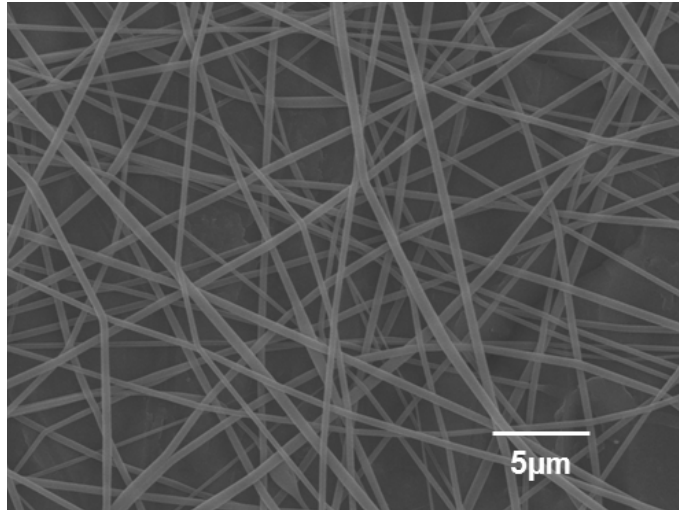

(a)

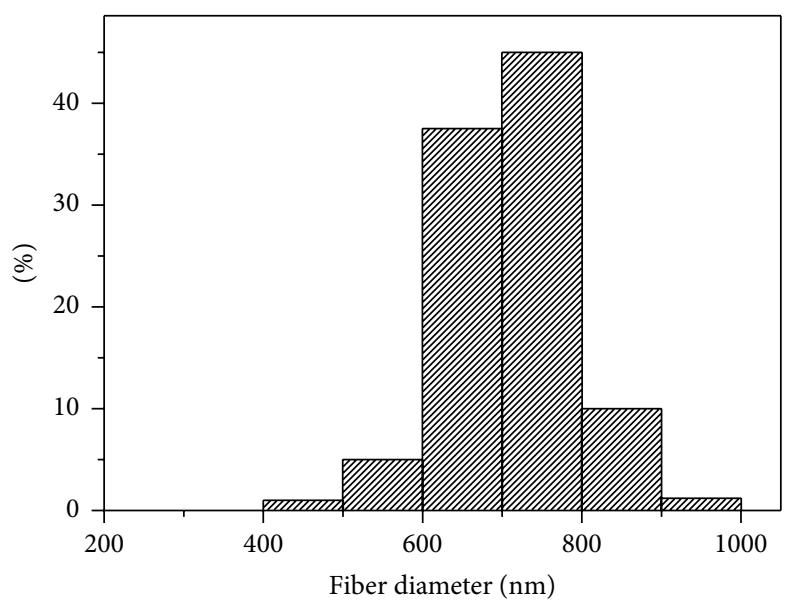

(b)

FIGURE 3: (a) SEM image and (b) diameter distribution of PEDOT:PSS/PVP nanofibers by conventional electrospinning.

So we prepared aligned nanofibers. So aligned fibers were prepared via centrifugal electrospinning [14]. Figure 2(a) shows the SEM image of aligned straight PEDOT:PSS/PVP nanofibers with diameter of about $800 \mathrm{~nm}$. $I-V$ curve of single PEDOT:PSS/PVP nanofiber was characterized (Figure 2(b)) and the resistivity was at the magnitude of $10^{5} \Omega \cdot \mathrm{m}$.

To increase the contact area with CO gas, PEDOT:PSS/ PVP nanofiber membrane was fabricated on the QCM by conventional electrospinning. Figure 3(a) shows the SEM image of the nanofiber membrane on the QCM. Each individual fiber is quite uniform in cross section. And the diameters of the fibers are 600-800 nm, as shown in Figure 3(b).

Figure 4 shows the Raman shifts of the electrospun PEDOT:PSS/PVP fibers. The band at $438 \mathrm{~cm}^{-1}$ is assigned to the $\mathrm{SO}_{2}$ bending. The band $576 \mathrm{~cm}^{-1}$ can be assigned to the oxyethylene ring deformation, the band $700 \mathrm{~cm}^{-1}$ to the symmetric C-S-C deformation, and $1099 \mathrm{~cm}^{-1}$ to the C$\mathrm{O}-\mathrm{C}$ deformation. The band at $1250 \mathrm{~cm}^{-1}$ can be assigned to the $\mathrm{C}-\mathrm{C}$ interring stretching, $1366 \mathrm{~cm}^{-1}$ to the $\mathrm{C}-\mathrm{C}$ stretching, $1430 \mathrm{~cm}^{-1}$ to the symmetric $\mathrm{C}=\mathrm{C}(-\mathrm{O})$ stretching, and $1529 \mathrm{~cm}^{-1}$ to the asymmetric $\mathrm{C}=\mathrm{C}$ stretching. The observed band positions and their assignments are in accord with published data [11].

3.2. Time Response to CO Gas. As is well known, the mechanism of QCM is based on Sauerbrey Equation (1)

$$
\Delta f=-2.26 \times 10^{-6} \times f_{0}^{2} \frac{\Delta m}{A},
$$

where $\Delta f$ is the measured frequency difference, $f_{0}=$ 7.995 MHz the original oscillation frequency of QCM, which 


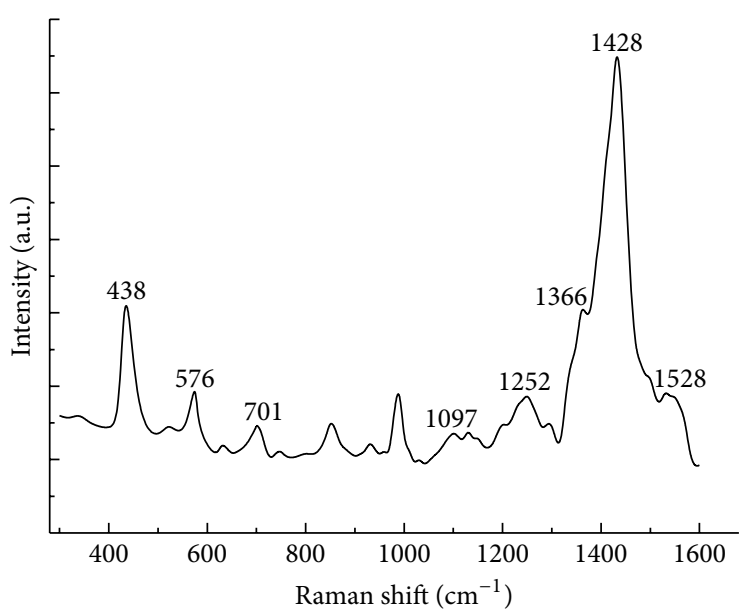

Figure 4: Raman shifts of the electrospun PEDOT:PSS/PVP fibers.

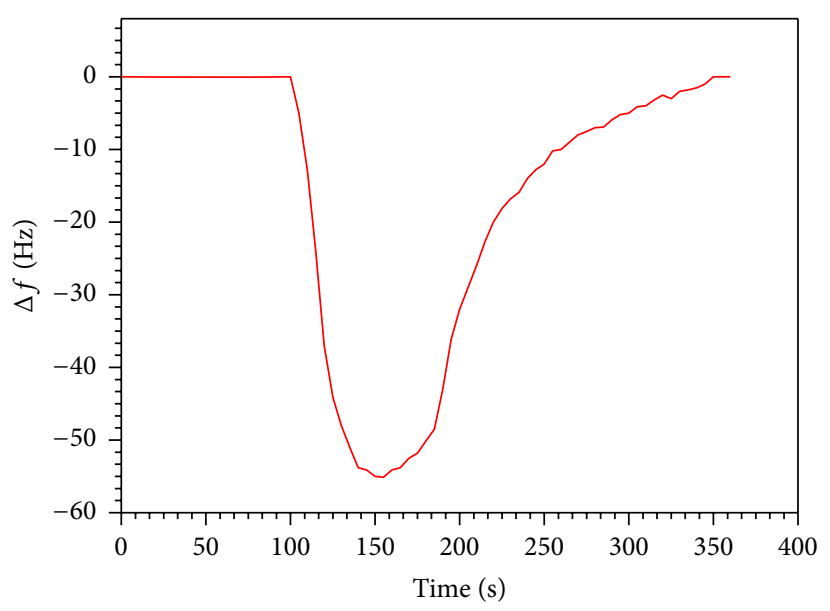

(a)

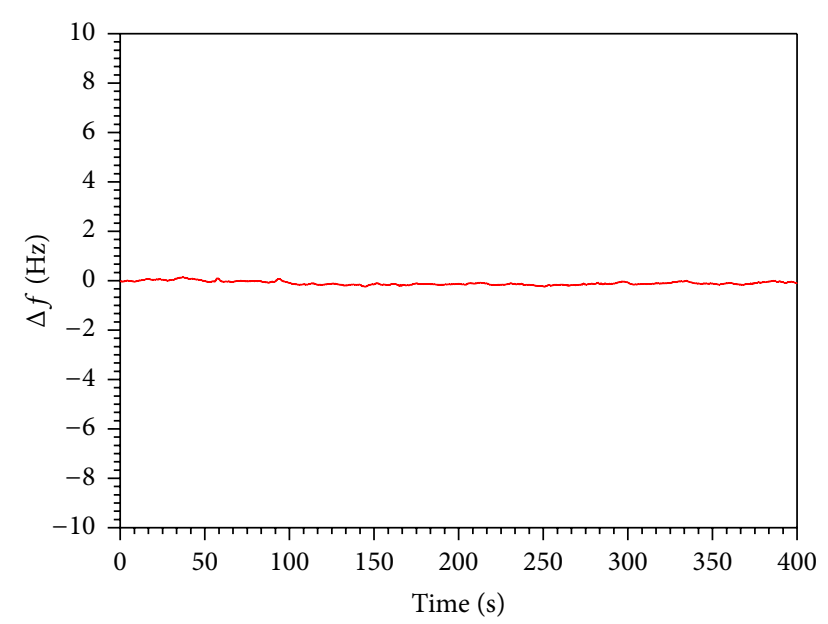

(b)

FIgUre 5: Response to $50 \mathrm{ppm}$ CO of the gas sensor based on PEDOT:PSS/PVP nanofibers (a) and pure PVP nanofibers (b).

is a constant, $\Delta m$ is the change in mass on the surface of the crystal, and $A=0.196 \mathrm{~cm}^{2}$ is the effective area of electrode. For this AT-cut quartz crystal, every hertz frequency change is equivalent to $1.34 \mathrm{ng}$, since Sauerbrey equation is suitable for materials which do not dissipate any energy during the oscillation, including metallic and metal oxides coatings. In other words, the relation remains invalid for soft or viscoelastic films, which do not completely couple with the oscillating crystal, thereby causing positive frequency shift and then an underestimation of mass. In our experiment, after the PEDOT:PSS/PVP nanofibers on QCM were exposed to different concentrations of $\mathrm{CO}$, respectively, the frequency difference remains negative, and the sensing layer acquires a stable structural conformation. This stability is most probably due to the establishment of a rigid three-dimensional network. For this reason, we neglected the viscoelasticity of the sensing layer and only molecular uptake is considered. So the Sauerbrey equation was used in the paper. According to the Sauerbrey formula, the frequency difference of the QCM is linearly related to mass change. Figure 5(a) shows that the frequency difference rapidly decreased as the $50 \mathrm{ppm}$ $\mathrm{CO}$ gas flowed into the test chamber and achieved stability after tens of seconds. Then when the $\mathrm{CO}$ valve was closed and $\mathrm{N}_{2}$ gas was filled into the chamber, the frequency difference increased until being back to zero $\mathrm{Hz}$. When $\mathrm{CO}$ gas flowed into the test chamber at $50 \mathrm{ppm}$, the maximum of frequency difference is about $-54 \mathrm{~Hz}$ as shown in Figure 5(a). Thus the mass change of PEDOT:PSS/PVP on the QCM could be calculated. According to (1) the mass change is $72.36 \mathrm{ng}$; that is to say, the PEDOT:PSS/PVP nanofibers adsorb $72.36 \mathrm{ng}$ $\mathrm{CO}$. The response was comparable to other gas sensors based on individual PEDOT:PSS nanofibers [15] because the PEDOT:PSS nanofibers have high surface-to-volume ratio in favor of contacting more $\mathrm{CO}$ molecules to increase the response.

To assess whether the $\mathrm{CO}$ adsorption was affected by PVP, we tested the QCM covered pure PVP nanofibers sensing to $50 \mathrm{ppm} \mathrm{CO}$. The results show that the frequency difference is at zero $\mathrm{Hz}$ (Figure 5(b)), which indicated that pure PVP nanofibers were not sensitive to CO. So the 


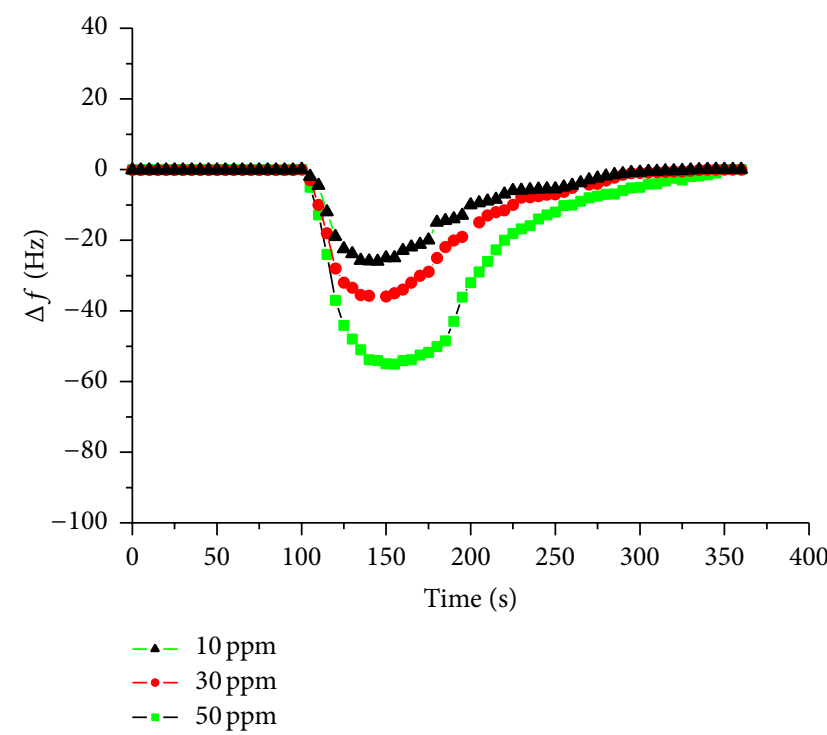

(a)

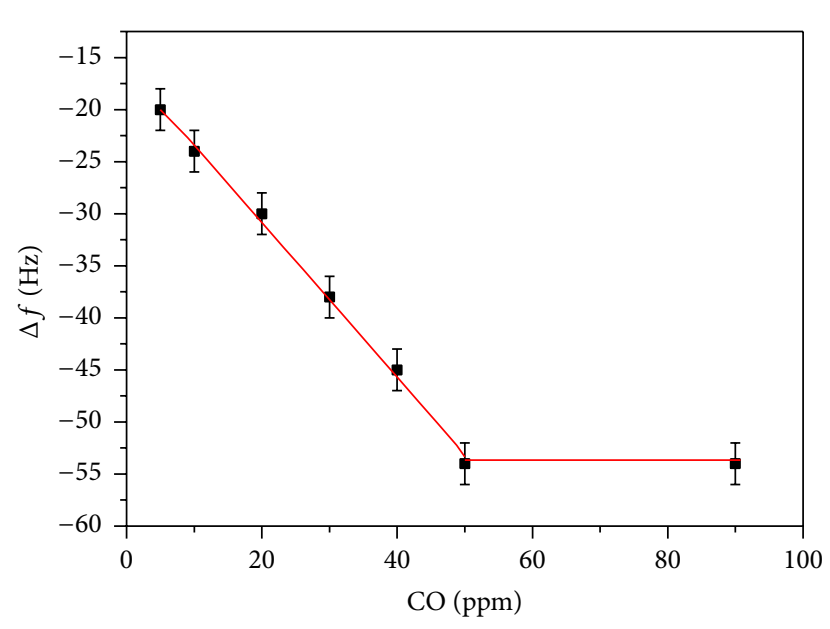

(b)

FIGURE 6: (a) The response characteristics of PEDOT:PSS/PVP nanofiber membrane sensing to 10, 30, and 50 ppm CO. (b) Relationship of frequency difference with $\mathrm{CO}$ concentration.

frequency difference decreased since the PEDOT:PSS in the fibers adsorbed the $\mathrm{CO}$ gas leading to the mass increasing of the nanofiber membrane. Moreover, the nanofibers have advantages of large aspect ratio and high surface-to-volume ratio in favour of more PEDOT:PSS contacting CO molecules and shortening the response time.

3.3. Gas Concentration Measurement. To obtain more details of the sensing information of the nanofibers, other $\mathrm{CO}$ concentrations were also measured. Figure 6(a) shows the response characteristics of the PEDOT:PSS/PVP nanofiber membrane sense to 10,30 , and $50 \mathrm{ppm}$ CO. It can be seen that the frequency difference increases with the concentration. Under the same flow rate conditions, more CO molecules enter the gas chamber with increased concentration, which enhanced the contact between CO and PEDOT:PSS/PVP. The adsorbed $\mathrm{CO}$ amount rises and the frequency difference increases.

In order to study the $\mathrm{CO}$ concentration detection limits, over $50 \mathrm{ppm} \mathrm{CO}$ was measured. The results show that the maximum frequency difference is similar to that of $50 \mathrm{ppm}$, which illustrates the adsorption of the nanofiber membrane for $\mathrm{CO}$ gas reached saturated at $50 \mathrm{ppm}$. Generally, the frequency difference by QCM method is proportional to the concentration of gas detected [16]. Figure 6(b) reveals a nearly linear relationship between the frequency difference and $\mathrm{CO}$ concentration less than $50 \mathrm{ppm}$ and the frequency difference reaches plateau after $50 \mathrm{ppm}$. The solid line is linear fitting to the data. If the thickness of the fiber membrane is different, the CO saturated concentration may be also not the same for the different contacting area to CO. Thus the sensor based on
PEDOT:PSS/PVP nanofibers by QCM method was suitable for the low concentration gas measurement.

According to the results previously reported [17, 18] and obtained in this work, the sensing mechanism of PEDOT:PSS/PVP nanofibers could be suggested: PEDOT/ PSS has a polar structure, PEDOT is the positive part, and PSS is the negative part. It shows a better adsorption to $\mathrm{CO}$ polar molecules, which leads to the reduced frequency difference of QCM. $\mathrm{N}_{2}$ gas had no effect on the fibers sensing because of its nonpolar structure. In the CO/PEDOT:PSS adsorption system, CO molecules play a role as the electron donor to PEODT:PSS, which leads to the reduction of holes. If the detection of $\mathrm{CO}$ occurs via the mechanism as shown in Figure 7, it shows the interaction between $\mathrm{CO}$ and PEDOT:PSS.

\section{Conclusions}

The PEDOT:PSS/PVP nanofibers were successfully fabricated on QCM via electrospinning. The results show that the PEDOT:PSS/PVP nanofiber membrane was sensitive to low concentration (5-50 ppm) CO gas. The frequency difference was proportional to the concentration of $\mathrm{CO}$. When the concentration increased to a certain value the adsorption of nanofiber membrane for $\mathrm{CO}$ reached saturation. The size effect of nanofibers makes the PEDOT:PSS/PVP more comprehensive not only on solar cells and electronic devices, but also on gas sensors.

\section{Competing Interests}

The authors declare that they have no competing interests. 


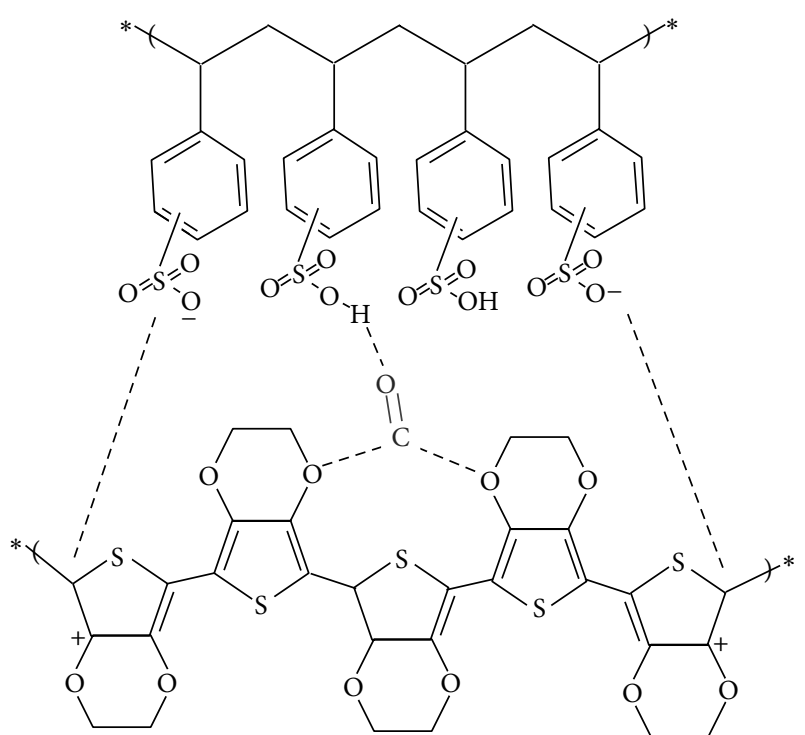

FIGURE 7: Schematic illustration of the sensing mechanism.* represents the degree of polymerization.

\section{Acknowledgments}

This work was supported by the National Natural Science Foundation of China (51373082), the Taishan Scholars Program of Shandong Province, China (ts20120528), and the Postdoctoral Scientific Research Foundation of Qingdao.

\section{References}

[1] H. Tang, M. Yan, H. Zhang et al., "A selective $\mathrm{NH}_{3}$ gas sensor based on $\mathrm{Fe}_{2} \mathrm{O}_{3}-\mathrm{ZnO}$ nanocomposites at room temperature," Sensors and Actuators B: Chemical, vol. 114, no. 2, pp. 910-915, 2006.

[2] M. Tonezzer and N. V. Hieu, "Size-dependent response of single-nanowire gas sensors," Sensors and Actuators B: Chemical, vol. 163, no. 1, pp. 146-152, 2012.

[3] J.-A. Park, J. Moon, S.-J. Lee, S. H. Kim, T. Zyung, and H. Y. Chu, "Structure and $\mathrm{CO}$ gas sensing properties of electrospun $\mathrm{TiO}_{2}$ nanofibers," Materials Letters, vol. 64, no. 3, pp. 255-257, 2010.

[4] Q. Zhou, C. Tang, S. P. Zhu, and W. G. Chen, "NiO doped $\mathrm{SnO}_{2}$ $\mathrm{p}-\mathrm{n}$ heterojunction microspheres: preparation, characterisation and CO sensing properties," Materials Technology, vol. 30, no. 6, pp. 349-355, 2015.

[5] C.-Y. Lin, J.-G. Chen, C.-W. Hu, J. J. Tunney, and K.-C. Ho, "Using a PEDOT:PSS modified electrode for detecting nitric oxide gas," Sensors and Actuators B: Chemical, vol. 140, no. 2, pp. 402-406, 2009.

[6] R. D. Ladhe, K. V. Gurav, S. M. Pawar, J. H. Kim, and B. R. Sankapal, "p-PEDOT:PSS as a heterojunction partner with n$\mathrm{ZnO}$ for detection of LPG at room temperature," Journal of Alloys and Compounds, vol. 515, pp. 80-85, 2012.

[7] C.-J. Chiang, K.-T. Tsai, Y.-H. Lee et al., "In situ fabrication of conducting polymer composite film as a chemical resistive $\mathrm{CO}_{2}$ gas sensor," Microelectronic Engineering, vol. 111, pp. 409-415, 2013.

[8] H. D. Zhang, C. C. Tang, Y. Z. Long et al., "High-sensitivity gas sensors based on arranged polyaniline/PMMA composite fibers," Sensors and Actuators A: Physical, vol. 219, pp. 123-127, 2014.

[9] C.-A. Dai, C.-J. Chang, H.-Y. Chi, H.-T. Chien, W.-F. Su, and W.-Y. Chiu, "Emulsion synthesis of nanoparticles containing PEDOT using conducting polymeric surfactant: synergy for colloid stability and intercalation doping," Journal of Polymer Science Part A: Polymer Chemistry, vol. 46, no. 7, pp. 2536-2548, 2008.

[10] P. Pacher, A. Lex, S. Eder et al., "A novel concept for humidity compensated sub-ppm ammonia detection," Sensors and Actuators B: Chemical, vol. 145, no. 1, pp. 181-184, 2010.

[11] J. Choi, J. Lee, J. Choi, D. Jung, and S. E. Shim, "Electrospun PEDOT:PSS/PVP nanofibers as the chemiresistor in chemical vapour sensing," Synthetic Metals, vol. 160, no. 13-14, pp. 14151421, 2010.

[12] E. Zampetti, S. Pantalei, A. Muzyczuk et al., "A high sensitive $\mathrm{NO}_{2}$ gas sensor based on PEDOT-PSS/TiO 2 nanofibres," Sensors and Actuators B: Chemical, vol. 176, pp. 390-398, 2013.

[13] H. Zeng, Y. Jiang, G. Xie, and J. Yu, "Polymer coated QCM sensor with modified electrode for the detection of DDVP," Sensors and Actuators B: Chemical, vol. 122, no. 1, pp. 1-6, 2007.

[14] S.-L. Liu, Y.-Z. Long, Z.-H. Zhang et al., "Assembly of oriented ultrafine polymer fibers by centrifugal electrospinning," Journal of Nanomaterials, vol. 2013, Article ID 713275, 9 pages, 2013.

[15] Y. Dan, Y. Cao, T. E. Mallouk, A. T. Johnson, and S. Evoy, "Dielectrophoretically assembled polymer nanowires for gas sensing," Sensors and Actuators, B: Chemical, vol. 125, no. 1, pp. 55-59, 2007.

[16] Y. Yang, Y. Jiang, J. Xu, and J. Yu, "PEDOT multilayer LB films and their gas sensitivity based on quartz crystal microbalance," Materials Science and Engineering B: Solid-State Materials for Advanced Technology, vol. 139, no. 2-3, pp. 251-255, 2007.

[17] G. A. Sotzing, S. M. Briglin, R. H. Grubbs, and N. S. Lewis, "Preparation and properties of vapor detector arrays formed from poly(3,4-ethylenedioxy)thiophene-poly(styrene sulfonate)/ insulating polymer composites," Analytical Chemistry, vol. 72, no. 14, pp. 3181-3190, 2000.

[18] R. Memarzadeh, H.-B. Noh, S. Javadpour, F. Panahi, A. Feizpour, and Y.-B. Shim, "Carbon monoxide sensor based on a B2HDDT-doped PEDOT: PSS layer," Bulletin of the Korean Chemical Society, vol. 34, no. 8, pp. 2291-2296, 2013. 

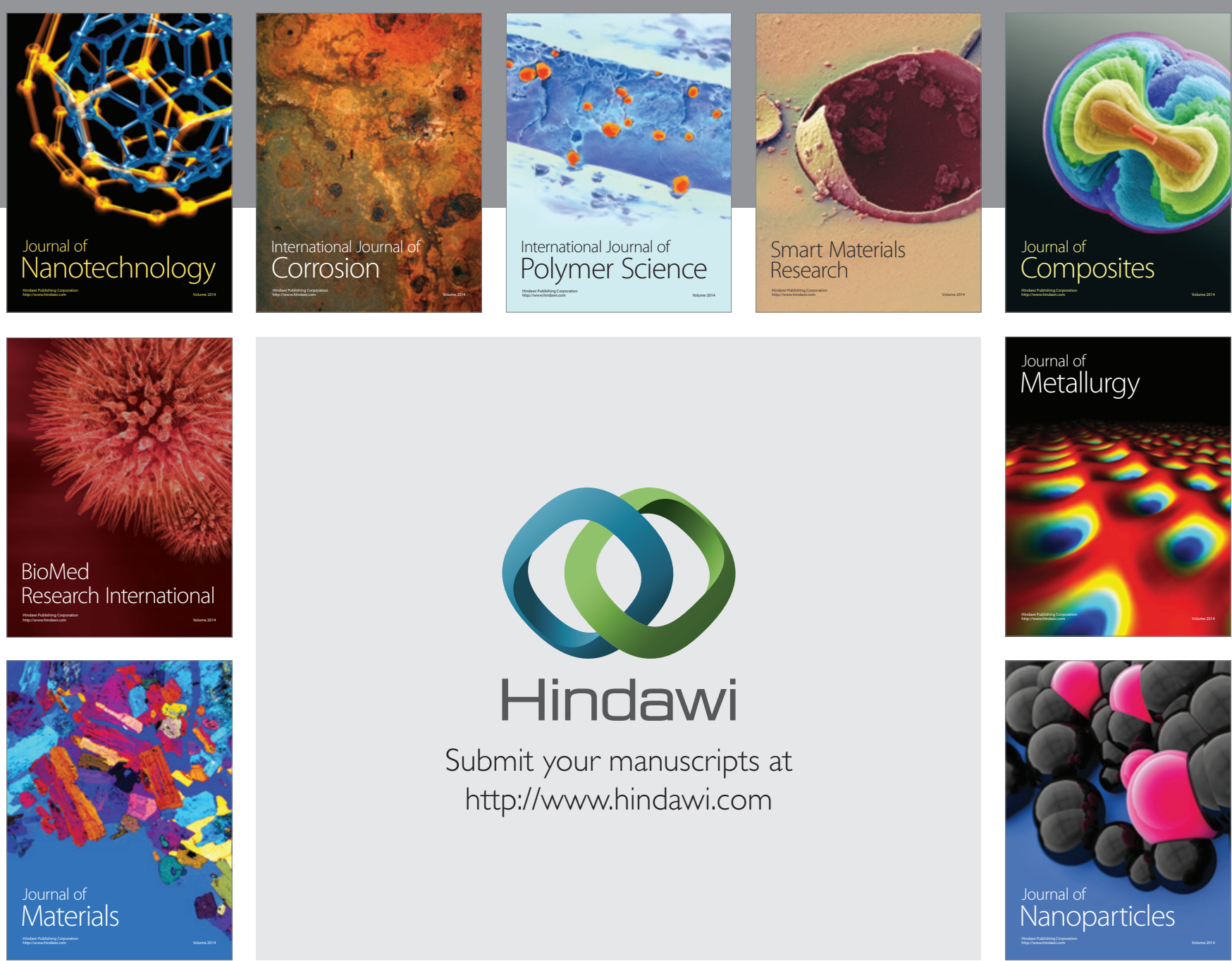

\section{Hindawi}

Submit your manuscripts at

http://www.hindawi.com

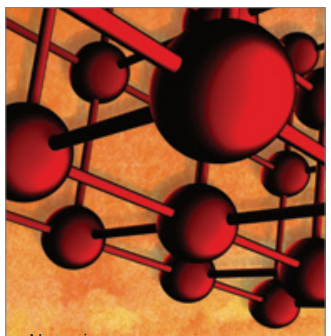

Materials Science and Engineering
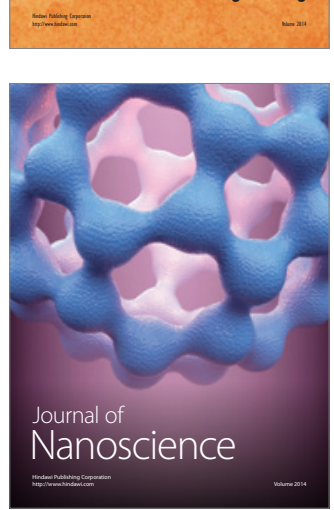
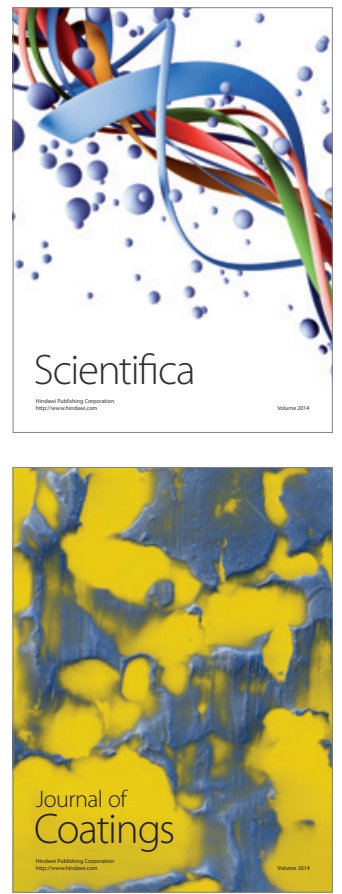
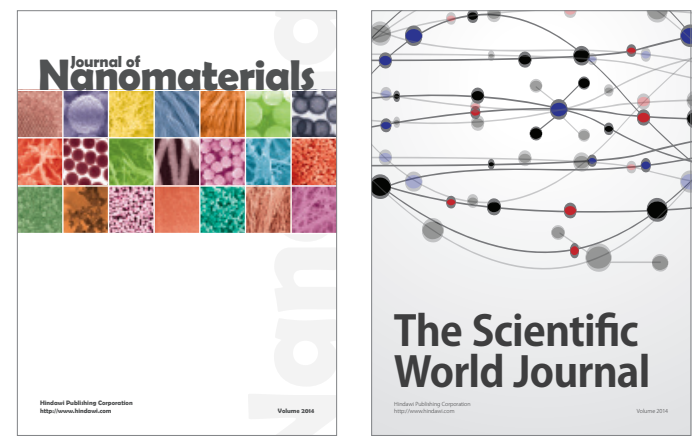

The Scientific World Journal
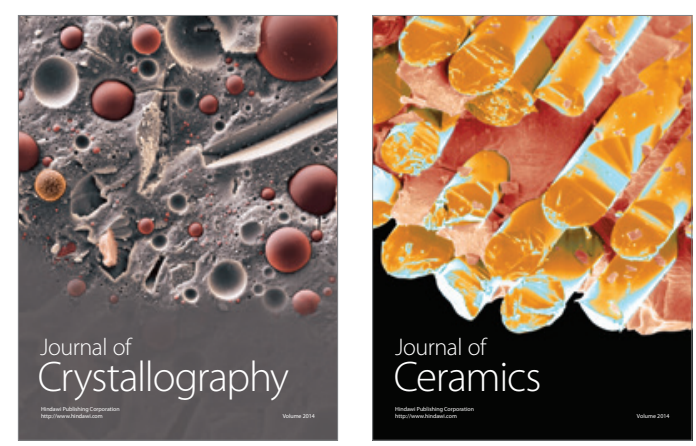
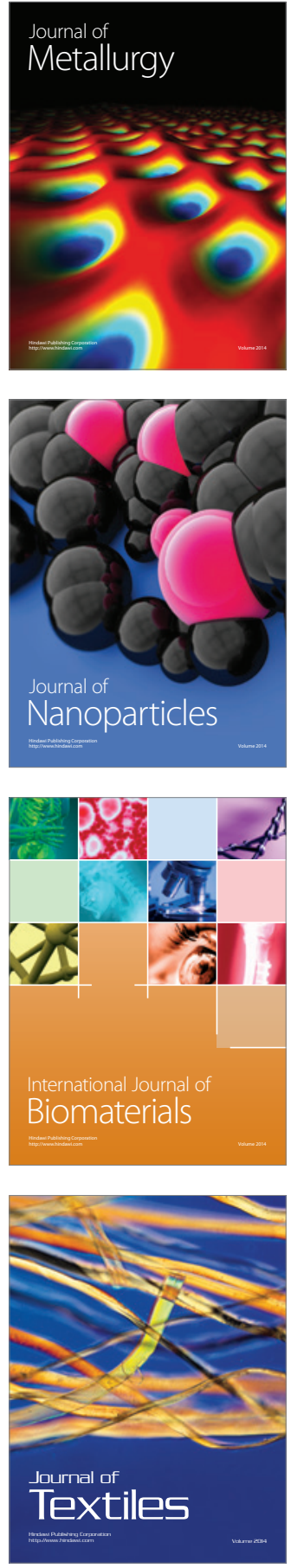